\title{
Alkalimetallderivate eines Alkoxysilazans
}

\author{
Michael Veith* und Jutta Böhnlein \\ Anorganische Chemie der Universität des Saarlandes, \\ D-6600 Saarbrücken 11
}

Eingegangen am 18. Oktober 1988

Keywords: Molecules with chelated metals/Unusual coordination numbers/Polycyclic compounds with alkali metals

In 1-tert-Butoxy-1,1,3,3,3-pentamethyldisilazan (1) läßt sich das Aminwasserstoffatom durch Alkalimetall-Atome ersetzen, was zu den Derivaten $2(\mathrm{Li}), 3(\mathrm{Na}), 4(\mathrm{~K})$ und $5(\mathrm{Rb})$ führt. Alle Verbindungen liegen in Lösung und im Kristall (bei 2 nicht untersucht) als Dimere vor, wobei 4 und 5 isotype Kristallstrukturen besitzen. Nach Röntgenstrukturanalysen unterscheidet sich die Struktur des Natriumderivates grundlegend von derjenigen der entsprechenden Kalium- bzw. Rubidiumverbindung: in 3 besteht der Tricyclus aus drei kantenverknüpften Vierringen $(2 \times \mathrm{NaNSiO}, 1 \times$ $\mathrm{Na}_{2} \mathrm{~N}_{2}$ ), die eine gemeinsame Ecke und die Punktsymmetrie $C_{2}$ (2) besitzen, während der Tricyclus in 4 ausschließlich aus kantenverknüpften Vierringen aufgebaut ist $\left(2 \times \mathrm{KNSiO}, 1 \times \mathrm{K}_{2} \mathrm{~N}_{2}\right)$, aber das Symmetrieelement $C_{i}(\overline{1})$ enthält. Im ersten Fall besitzen die beiden Natriumatome unterschiedliche Koordinationszahlen $(\mathrm{KZ}=4$ und 2$)$, im zweiten Fall sind die Kaliumatome gleichartig $(\mathrm{KZ}=3)$. Erwartungsgemä $\mathrm{B}$ kommt dem niedriger koordinierten Natriumatom $\mathrm{Na}(2)$ ein kürzerer Abstand zum Stickstoff zu als dem tetraedrisch koordinierten $\mathrm{Na}(1) \mathrm{Na}(1)-\mathrm{N} 2.467(5)$, Na(2)-N 2.399(5) \&]. Die Abstände des Stickstoff- und Sauerstoffatoms zum $K$ in 4 sind lang und können mit der ungewöhnlichen trigonal pyramidalen Koordination um das Metallatom erklärt werden. Die ${ }^{1} \mathrm{H}-\mathrm{NMR}$-Spektren der Toluollösungen von 2-5 deuten auf Fluktuationen innerhalb der Moleküle hin.

Bis(amino)silane und Oxoaminosilane haben sich in der Vergangenheit als vorteilhaft für die Stabilisierung niedriger Koordinationszahlen an Metallatomen erwiesen ${ }^{11}$. Speziell Alkalimetalle lassen sich mit den Ligandensystemen A, B, C und $\mathbf{D}$ in ungewöhnlicher Koordinationssphäre erhalten.

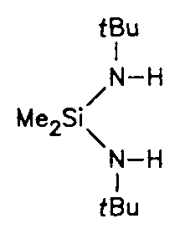

A
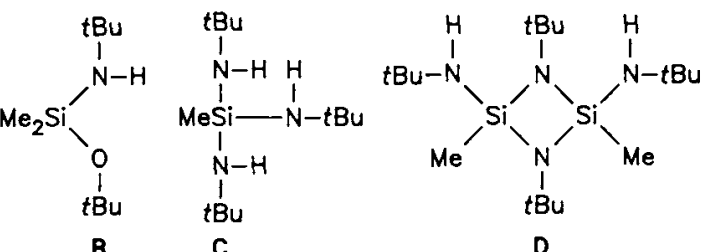

Tauscht man die Wasserstoffatome in A durch Lithiumatome aus, so bildet sich ein Dimeres, in dem ein $\mathrm{Li}_{4} \mathrm{~N}_{4^{-}}$ Antiprisma mit trigonal-pyramidalen Lithiumatomen als zentrales Strukturelement auftritt ${ }^{2}$. Auch die Lithium- und Natriumderivate von $\mathbf{B}$ sind jeweils dimer ${ }^{3,4}$. Das Trilithiumsalz von $\mathbf{C}$ existiert wiederum nur als das Doppelte der Formel, wobei die Lithiumatome einen stark gefalteten Sechsring bilden, der von den Stickstoffatomen koordinativ ergänzt wird $^{\text {s) }}\left[\mathrm{C}_{3}\right.$-Symmetrie, analoge Strukturen findet man bei $t \mathrm{Bu}-\mathrm{Si}(\mathrm{N} t \mathrm{BuLi})_{3}$ und $\left.\mathrm{Ph}-\mathrm{Si}(\mathrm{N} t \mathrm{BuLi})_{3}{ }^{2}{ }^{2}\right]$. Das Di-

\section{Alkali Metal Derivatives of an Alkoxysilazane}

The hydrogen atom of 1-tert-butoxy-1,1,3,3,3-pentamethyldisilazane (1) can be replaced by alkali atoms yielding the derivatives $2(\mathrm{Li}), 3(\mathrm{Na}), 4(\mathrm{~K})$, and $5(\mathrm{Rb})$. All molecules are dimeric in solution as well as in the crystal (not investigated for 2), 4 and 5 forming isotypic crystal structures. According to X-ray structure analysis the structure of the sodium derivative is fundamentally different from that of the corresponding potassium and rubidium one: the tricyclic system in 3 consists of three four-membered rings sharing edges and one common corner $\left(2 \times \mathrm{NaNSiO}, 1 \times \mathrm{Na}_{2} \mathrm{~N}_{2}\right)$, and belongs to the point symmetry $C_{2}(2)$, while the tricycle in 4 has exclusively edge-sharing four-membered rings $(2 \times \mathrm{KNSiO}$, $1 \times \mathrm{K}_{2} \mathrm{~N}_{2}$ ) and has a $C_{i}(\overline{1})$ symmetry element. In the first case the two sodium atoms have different coordination numbers (c.n. $=4$ and 2 ), in the second one the two potassium atoms are alike $(c . n .=3)$. As expected, the lower coordinated sodium atom $\mathrm{Na}(2)$ exhibits shorter distances to the nitrogen than the tetrahedrally coordinated $\mathrm{Na}(1)[\mathrm{Na}(1)-\mathrm{N} 2.467(5), \mathrm{Na}(2)-\mathrm{N} 2.399(5) \AA]$ ]. The distances from the oxygen and nitrogen atom to potassium within 4 are long, which can be attributed to the unusual trigonal pyramidally coordinated metal atom. The ${ }^{1} \mathrm{H}-\mathrm{NMR}$ spectra of toluene solutions of $2-5$ are consistent with bond fluctuation within the molecules.

lithiumsalz von $\mathbf{D}$ schließlich ist monomer, wobei als zentraler Polycyclus ein verzerrter $\mathrm{Si}_{2} \mathrm{~N}_{4} \mathrm{Li}_{2}-\mathrm{Kubus}$ auftritt ${ }^{6}$. Auch in diesem Molekül besitzen die Lithiumatome eine trigonal-pyramidale Koordinationssphäre.

Es hat uns interessiert, wie sich die Struktur eines Silazans beim Übergang von leichten Alkalielementen zu schwereren ändert. Da die obigen Aminosilane A-D sich nur schwer mit höheren Alkalielementen umsetzen, sind wir auf ein basischeres Amin ausgewichen. Wir haben dabei den Liganden B so modifiziert, da $B$ wir an Stelle der tert-Butylgruppe am Stickstoffatom eine Trimethylsilylgruppe einbauten. Im folgenden beschreiben wir die Synthese dieses Liganden, seine Derivatisierung durch Austausch des Wasserstoffatoms durch $\mathrm{Li}, \mathrm{Na}, \mathrm{K}$ und $\mathrm{Rb}$ und die strukturellen Aspekte, die sich beim Vergleich der Derivate ergeben.

Die Darstellung des Alkoxysilazans 1 erfolgte nach klassischen Methoden ${ }^{7)} ; 1$ kann in drei Reaktionsschritten aus Dichlordimethylsilan, Natrium-tert-butylat, Ammoniak und Chlortrimethylsilan entsprechend Gl. (1) erhalten werden.

$\mathrm{Me}_{2} \mathrm{SiCl}_{2}+t \mathrm{BuONa} \rightarrow \mathrm{Me}_{2} \mathrm{SiCl}(\mathrm{O} t \mathrm{Bu})+\mathrm{NaCl}$

$\mathrm{Me}_{2} \mathrm{SiCl}(\mathrm{O} t \mathrm{Bu})+2 \mathrm{NH}_{3} \rightarrow \mathrm{Me}_{2} \mathrm{Si}(\mathrm{O} t \mathrm{Bu})\left(\mathrm{NH}_{2}\right)+\mathrm{NH}_{4} \mathrm{Cl}$

$\mathrm{Me}_{2} \mathrm{Si}(\mathrm{O} t \mathrm{Bu})\left(\mathrm{NH}_{2}\right)+\mathrm{ClSiMe}_{3}+\mathrm{Et}_{3} \mathrm{~N} \rightarrow$

$$
\mathrm{Me}_{2} \mathrm{Si}(\mathrm{O} t \mathrm{Bu}) \mathrm{NH}\left(\mathrm{SiMe}_{3}\right)+\mathrm{Et}_{3} \mathrm{NHCl}
$$

1 
Das Silazan 1 kann über Destillation als reine, farblose Flüssigkeit in etwa $60 \%$ Ausbeute isoliert werden. Das ${ }^{1} \mathrm{H}$ NMR-Spektrum weist zwei intensitätsgleiche Signale für tert-Butyl- und Trimethylsilylgruppe und ein um $1 / 3 \mathrm{klei}$ neres Signal für die Dimethylsilylgruppe auf.

Die Überführung dieses Silazans in die Alkalimetallderivate $\mathbf{2}-\mathbf{5}$ kann auf drei verschiedenen Wegen erfolgen [G1. $(2)]^{7)}$, wobei die "Styrol-Methode“ am allgemeinsten anwendbar ist.

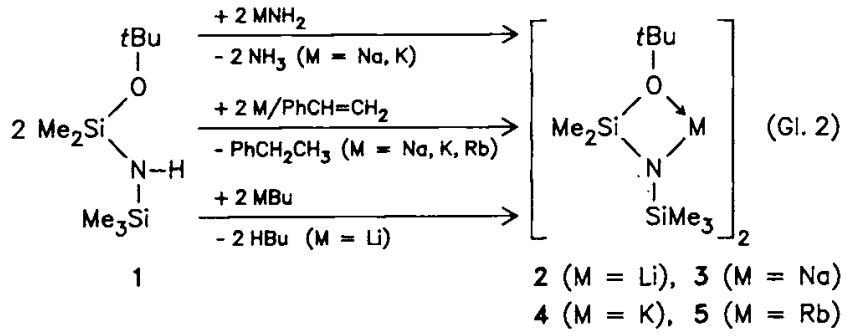

Die Alkaliderivate des Silazans 1 lassen sich durch Kristallisation aus Toluollösungen oder durch Sublimation erhalten. Die Lithiumverbindung 2 sublimiert in stark verwachsenen, sehr kleinen Nädelchen, die für Einkristallbeugung ungeeignet sind. Wie die Elementaranalysen belegen, wird im Kristall kein Lösungsmittel eingebaut. In den Massenspektren, bei den Molmassenbestimmungen und in den Strukturanalysen (siehe folgendes Kapitel) findet man immer das Doppelte der einfachen Masse, also dimere Moleküle.

Die ${ }^{1} \mathrm{H}$-NMR-Spektren der Verbindungen 2, 3, 4 und 5 sind sich untereinander sehr ähnlich und bestehen wie bei 1 nur aus drei Signalen. Hält man sich die im Kristall gefundenen Strukturen vor Augen (siehe weiter unten), so sollte man, unabhängig vom jeweiligen Strukturtyp, wegen der chemischen Ungleichheit zwei Signale für die Methylgruppen am Silicium finden. Selbst bei $-80^{\circ} \mathrm{C}$ treten im Spektrum von 3 und $4\left(-73^{\circ} \mathrm{C}\right)$ jedoch weiterhin nur drei und nicht vier Linien auf. Da Dissoziationen der dimeren Moleküle in monomere aufgrund der Molmassenbestimmungen weitgehend auszuschließen sind und wir auch bei geringen und hohen Konzentrationen der Metallsilazane keine Änderungen in den Spektren beobachtet haben, scheint die Entartung der Methylsilylsignale auf einem intramolekularen Proze $\mathrm{zu}$ beruhen. Wie bei anderen Alkoxysilazanen und Aminosilazanen von Elementen der dritten Hauptgruppe, bei denen wir diese intramolekularen Basenaustauschreaktionen im Detail studiert haben ${ }^{4,8)}$, scheinen auch bei 2, 3, 4 und 5 fluktuierende Systeme vorzuliegen. Weitergehende Untersuchungen an 3 und 4 legen nahe ${ }^{9}$, da $B$ offenbar ein Gleichgewicht zwischen den für das Natriumderivat und für das Kaliumderivat gefundenen Strukturty-

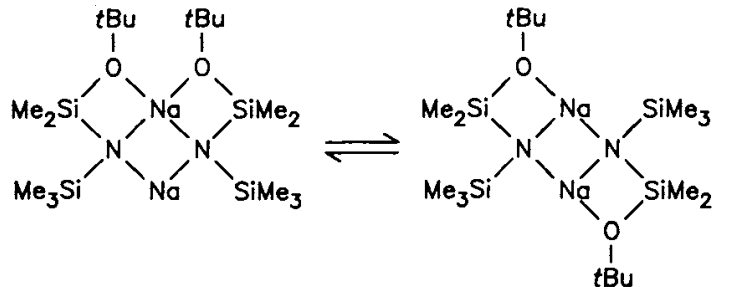

pen in Lösung auftritt. Da die Umwandlung der Strukturtypen (siehe unten) ineinander offenbar sehr schnell erfolgt, kann bis $-80^{\circ} \mathrm{C}$ kein „Ausfrieren“ einer Form realisiert werden. In Gl. (3) ist das oben angesprochene Gleichgewicht für 3 schematisch dargestellt.

In Tab. 1 sind die Einkristalldaten zusammen mit den wichtigsten experimentellen Parametern der Strukturbestimmung für die Verbindungen 3 und 4 aufgeführt. Zur Berechnung der Strukturamplituden wurden die Atomformfaktoren des SHELX-Systems verwendet ${ }^{10)}$, oder im Falle von Natrium und Kalium diejenigen der

Tab. 1. Kristalldaten und Daten zur Strukturbestimmung für die Verbindungen 3 und 4

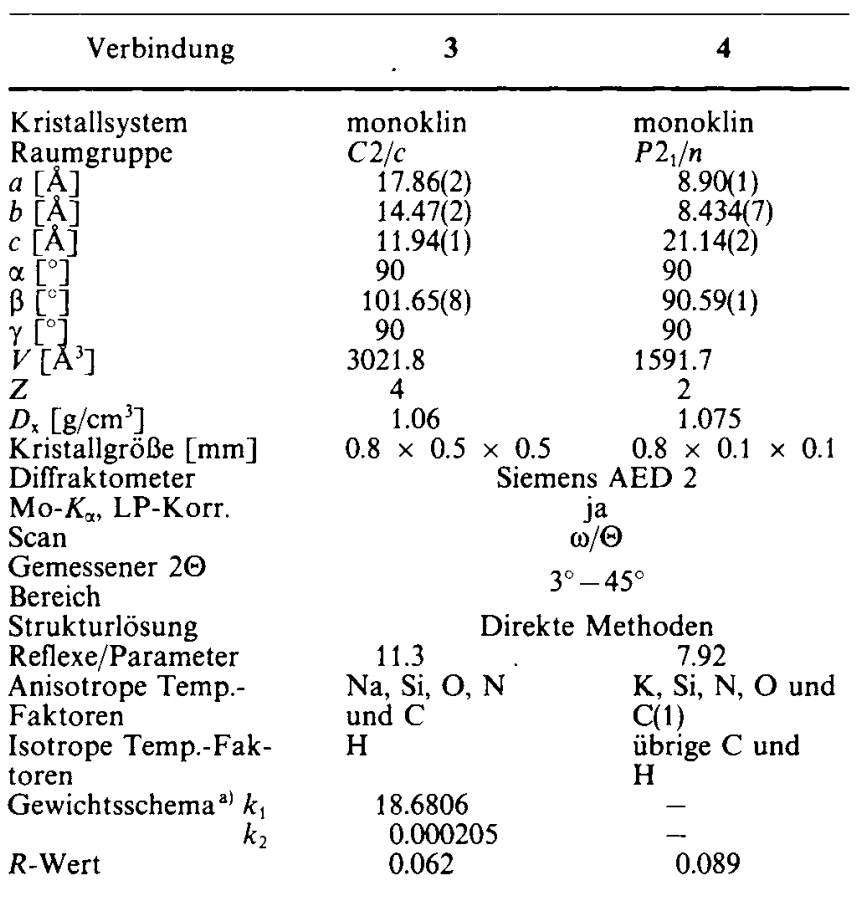

a) $k_{1}\left(\sigma_{\mathrm{F}}^{2}+k_{2} F^{2}\right)^{-1}$.

Tab. 2. Einige Bindungslängen $[\AA]$ und Winkel $\left[{ }^{\circ}\right.$ in $3^{a)}$

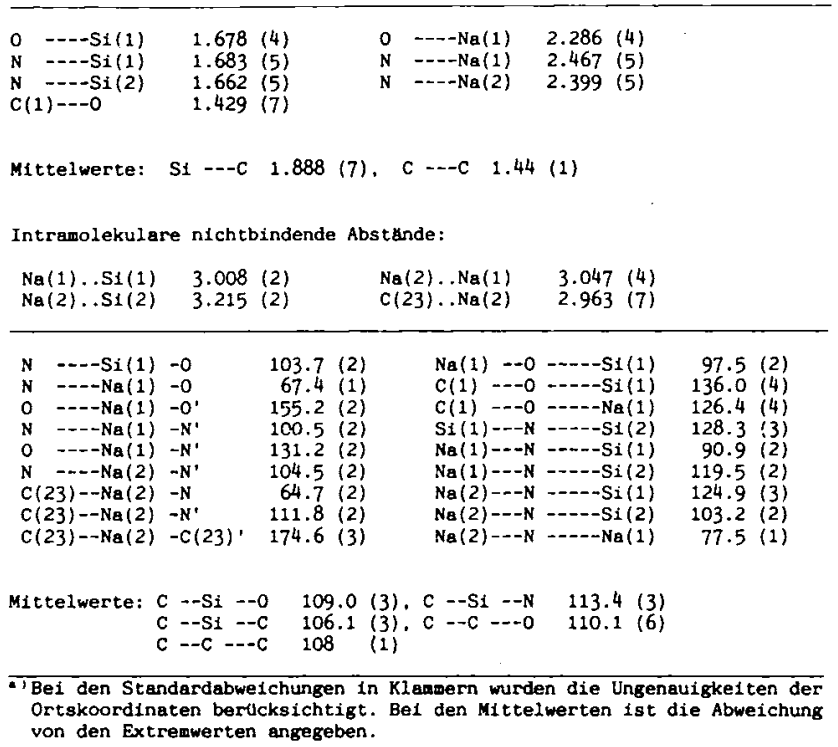


Tab. 3. Einige Bindungslängen $[\AA]$ und Winkel $\left[{ }^{\circ}\right]$ in $4^{\text {a) }}$

\begin{tabular}{|c|c|c|c|c|c|}
\hline 0 - - - - K & 2.71 & (1) & $N=--1-\operatorname{Si}(1)$ & 1.65 & (1) \\
\hline N $-\cdots---K$ & 2.87 & (1) & $\mathrm{N}------\mathrm{Si}(2)$ & 1.68 & (1) \\
\hline$N^{0}-1-2--k$ & 2.76 & (1) & $c(1)-\cdots-0$ & 1.42 & (2) \\
\hline & & & & & \\
\hline
\end{tabular}

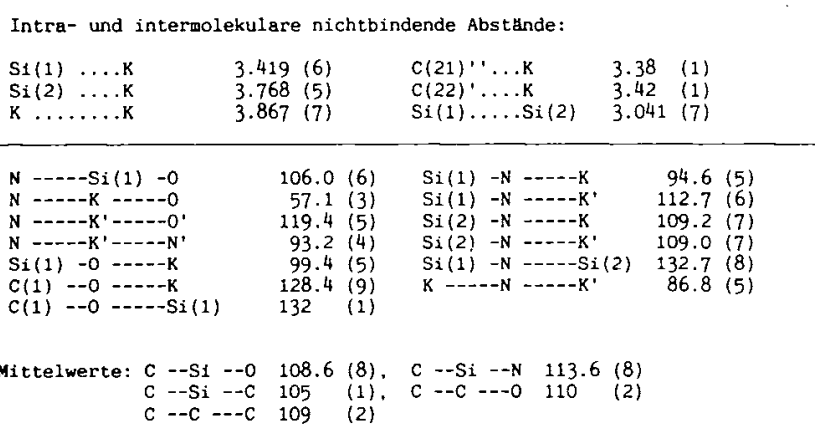

- Tei den Standardabweichungen in Klammern wurden die Ungenauigkeiten der Ortskoordinaten berucksichtigt. Bei den Mittelwerten ist die Abweichung von den Extrewwerten angegeben.

Tab. 4. Die Ortskoordinaten und der Parameter des Debye-WallerFaktors für die Atome der asymmetrischen Einheit von 3 und 4. Die $B$-Werte des Temperaturfaktors der Form $\exp \left(-B \cdot \sin ^{2} \Theta / \lambda\right)$ wurden nach Hamilton ${ }^{23)}$ aus den $u_{i j}$-Werten der Atome ${ }^{(2)}$ berechnet

\begin{tabular}{lcccc}
\hline \multicolumn{3}{l}{ Yerbindune 3} \\
\hline
\end{tabular}

Verbindung 4

\begin{tabular}{lcccc}
\hline & & $y$ & $z$ & $B\left[A^{2}\right]$ \\
Atom & $x$ & $y$ & & \\
$K$ & $0.6283(4)$ & $0.1756(5)$ & $0.5233(2)$ & $4.3(2)$ \\
$S_{1}(1)$ & $0.3185(5)$ & $0.0981(7)$ & $0.6124(2)$ & $4.4(3)$ \\
$S_{i}(2)$ & $0.2177(5)$ & $0.2263(6)$ & $0.4846(3)$ & $4.4(3)$ \\
0 & $0.484(1)$ & $0.179(1)$ & $0.6353(5)$ & $5.0(6)$ \\
$N$ & $0.314(1)$ & $0.110(1)$ & $0.5348(6)$ & $3.8(7)$ \\
$C(1)$ & $0.536(2)$ & $0.237(2)$ & $0.694(1)$ & $6 .(1)$ \\
$C(11)$ & $0.503(3)$ & $0.125(3)$ & $0.748(1)$ & $8.3(6)$ \\
$C(12)$ & $0.481(3)$ & $0.401(3)$ & $0.706(1)$ & $9.7(7)$ \\
$C(13)$ & $0.704(2)$ & $0.250(3)$ & $0.687(1)$ & $8.7(6)$ \\
$C(21)$ & $0.293(2)$ & $0.433(2)$ & $0.482(1)$ & $7.4(5)$ \\
$C(22)$ & $0.225(2)$ & $0.150(3)$ & $0.4029(9)$ & $7.4(5)$ \\
$C(23)$ & $0.009(2)$ & $0.249(2)$ & $0.500(1)$ & $6.6(5)$ \\
$C(3)$ & $0.163(2)$ & $0.193(3)$ & $0.655(1)$ & $7.1(5)$ \\
$C(4)$ & $0.321(2)$ & $-0.114(2)$ & $0.6382(9)$ & $6.3(5)$ \\
& & & &
\end{tabular}

Int. Tables ${ }^{11)} . F_{\mathrm{o}} / F_{\mathrm{c}}$-Vergleiche sowie anisotrope Temperaturfaktoren sind hinterlegt ${ }^{12)}$, während die Atomkoordinaten sowie isotrope Temperaturfaktoren in Tab. 4 enthalten sind. Wichtige Bindungslängen und -winkel sind in den Tab. 2 und 3 zusammengestellt.

In den Abb. 1 und 2 sind die Molekülstrukturen der Alkalimetallamide 3 und 4 dargestellt. Die Kristallstrukturen von 3 und 4 enthalten isolierte, polycyclische Einheiten, die aus dem Doppelten der einfachen Summenformel bestehen. In dem Natriumderivat 3 bilden die Atome $\mathrm{Na}, \mathrm{N}, \mathrm{O}$ und
Si einen Tricyclus, der wie folgt beschrieben werden kann: an einen planaren $\mathrm{Na}_{2} \mathrm{~N}_{2}$-Ring sind an benachbarten Kanten zwei weitere Vierecke, bestehend aus $\mathrm{O}-\mathrm{Si}-\mathrm{N}-\mathrm{Na}$ Einheiten, einmal ober- und einmal unterhalb der zentralen Ebene gebunden. Die Stickstoffatome besitzen als weitere Liganden Trimethylsilylreste, während an die Sauerstoffatome noch tert-Butylgruppen gebunden sind. Die Natriumatome nehmen in der Kristallstruktur spezielle Lagen ein. Ihre Positionen auf einer zweizähligen Achse bedingen für das Molekül die Punktsymmetrie $C_{2}$. Durch diese Anordnung sind die beiden Metallatome einander nicht äquivalent, sondern besitzen eine unterschiedliche Umgebung. So erreicht $\mathrm{Na}(1)$ eine verzerrt tetraedrische Koordination durch zwei Sauerstoff- und zwei Stickstoffatome des tertButoxy-trimethylsilyl-dimethylsilans, wie aus den Winkel-

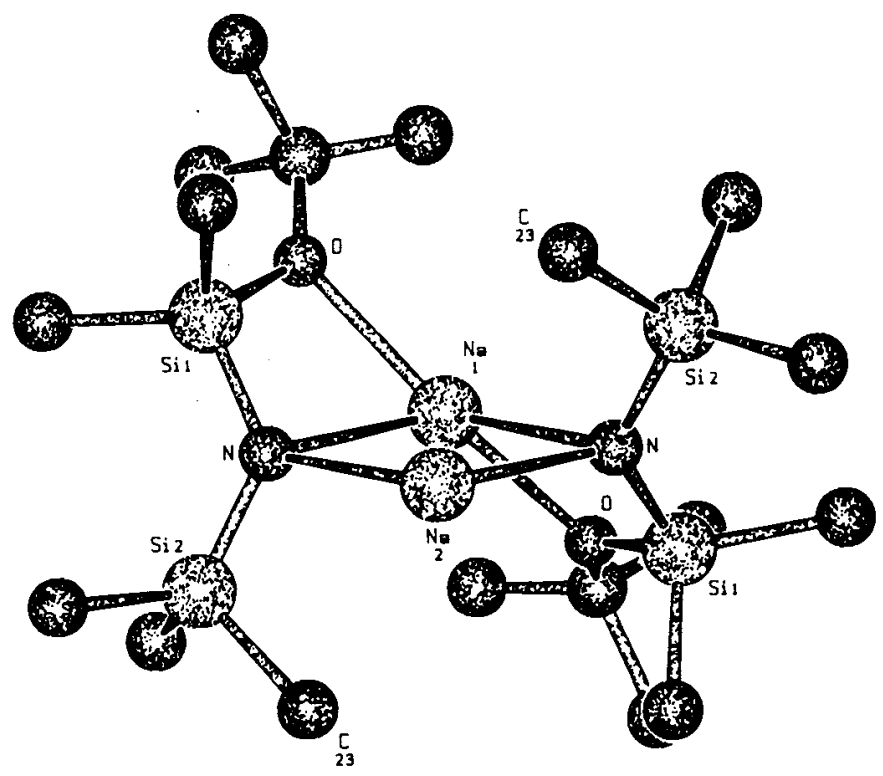

Abb. 1. Zeichnerische Wiedergabe der Molekülstruktur von 3 nach Röntgenstrukturanalyse

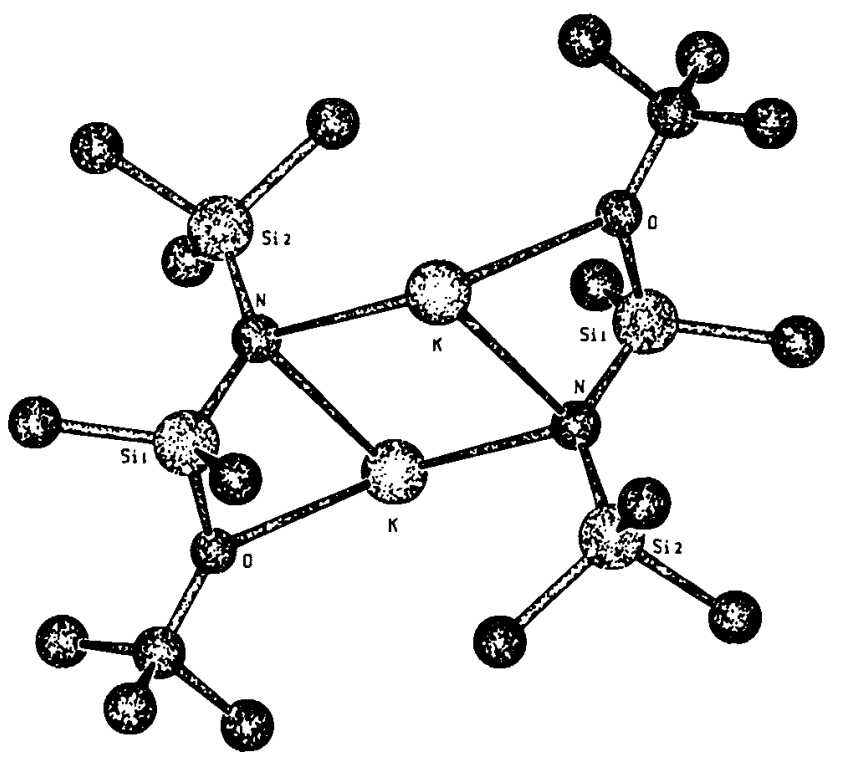

Abb. 2. Die Molekülstruktur von 4 
werten von Tab. 2 zu ersehen ist. Der auffallend große Wert für den $\mathrm{O}-\mathrm{Na}(1)-\mathrm{O}^{\prime}$-Winkel ist aus sterischen Gründen notwendig, um dem Raumbedarf der beiden voluminösen tert-Butylgruppen gerecht zu werden. $\mathrm{Na}$ (2) dagegen erfährt durch die beiden Stickstoffatome des Liganden nur eine zweifache Koordination. Geometrische Gründe sind für das Abweichen des $\mathrm{N}-\mathrm{Na}(2)-\mathrm{N}^{\prime}$-Winkels von der Linearität verantwortlich.

Unterschiedliche Koordinationszahlen an Natriumatomen in einer molekularen Einheit sind in der Literatur nur wenig bekannt ${ }^{13)}$. Untersucht man die Umgebung des niederkoordinierten Metallatoms in 3 näher, so stellt man fest, $\mathrm{da} ß \mathrm{Na}(2)$ zu den benachbarten Kohlenstoffatomen C(23) und $\mathrm{C}(23)^{\prime}$ kurze Abstände (2.963 $\AA$ ) aufweist. Im Vergleich mit Literaturwerten ${ }^{13.14}$ muß man jedoch von schwachen Wechselwirkungen ausgehen, die wohl mehr aus den sterischen Gegebenheiten des Molekülaufbaus als aus elektronischen Gründen erwachsen. Abb. 3 zeigt anhand des Kalottenmodells von 3, daß das Metallatom $\mathrm{Na}(2)$ trotz der benachbarten Atome $C(23)$ und $C(23)^{\prime}$ nur wenig abgeschirmt ist, was Auswirkungen auf die Reaktivität des Moleküls haben sollte" ${ }^{9}$. Vergleicht man die $\mathrm{Na}-\mathrm{N}$-Abstände miteinander, so kann man nicht zwischen einer formalen Zwei-Elektronen-Zwei-Zentren- und einer Zwei-Elektronen-Donorbindung unterscheiden. Die gefundenen Werte spiegeln lediglich die unterschiedlichen Koordinationszahlen der Metallatome wider. So ist der Abstand für das zweifach koordinierte $\mathrm{Na}(2)$ mit $2.399 \AA$ etwas kürzer als für das vierfach koordinierte $\mathrm{Na}(1)$ mit $2.467 \AA$. Ein Vergleich mit bereits bekannten Verbindungen ${ }^{15.16)}$ zeigt, $\mathrm{da} \mathrm{Na}-\mathrm{N}$ - und $\mathrm{Na}-\mathrm{O}$-Bindungsabstände im Bereich der Literaturwerte liegen. Der kurze Metall-Metall-Abstand von $3.047 \AA$ ist beim Zugrundelegen von ionischen Verhältnissen nicht ungewöhnlich. So findet man z. B. im $\mathrm{NaOCH}_{3}$ einen $\mathrm{Na}-\mathrm{Na}$ Abstand von $3.07 \AA^{177}$.

Beim Übergang vom Natrium- 3 zum Kaliumderivat 4 tritt ein signifikanter Sprung in den strukturellen Gegebenheiten auf. Es zeigt sich, daß die Verbindung 4 im Kristall als zentrosymmetrisches Dimer vorliegt. Die Entstehung des

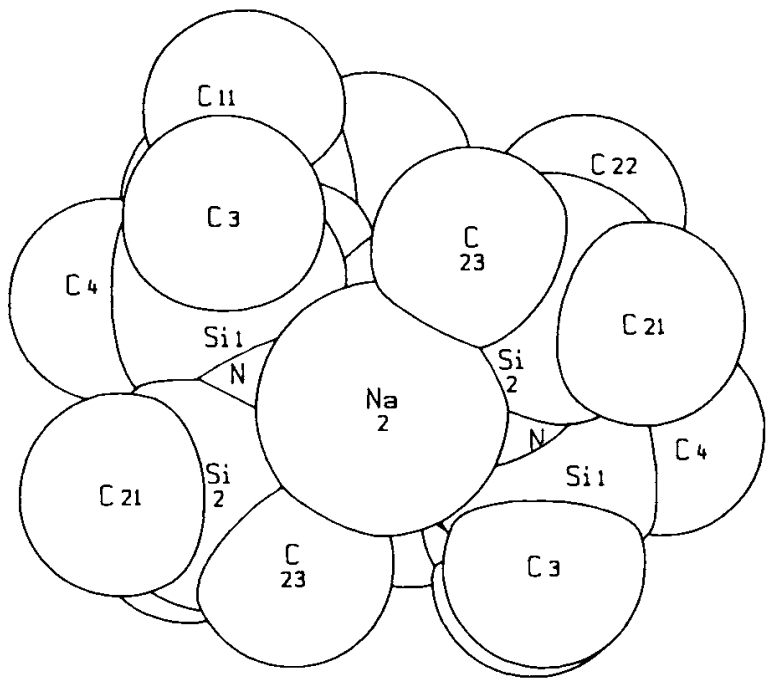

Abb. 3. Zeichnerische Darstellung von 3 mit van-der-Waals-Radien. Das Natriumatom $\mathrm{Na}(2)$ ist relativ wenig abgeschirmt
Tricyclus läßt sich durch die Vereinigung zweier Vierringstrukturelemente, bestehend aus $\mathrm{N}-\mathrm{Si}-\mathrm{O}-\mathrm{K}$, erklären, wobei die Stickstoffatome der beiden Untereinheiten wechselseitig als basische Elektronendonatoren gegenüber den Kaliumatomen wirken. Die drei derart an den Kanten verknüpften Vierringe stehen mit $131.0^{\circ}$ nur annähernd senkrecht aufeinander und bilden eine treppenartige Struktur. In dieser Anordnung erreicht jedes Kaliumatom eine recht ungewöhnliche Dreifachkoordination mit Winkeln zwischen $57^{\circ}$ und $119^{\circ}$. Infolge der geometrischen Zwänge ist sie weit entfernt von der idealen Symmetrie einer trigonal-planaren Figur. Aufgrund der beiden unterschiedlich langen Bindungsabstände $\mathrm{K}-\mathrm{N}$ von $2.87 \AA$ und $\mathrm{K}-\mathrm{N}^{\prime}$ von $2.76 \AA$ kann man das Gerüst von 4 auch als einen Achtring mit zwei zusätzlichen Brücken beschreiben. Die Bindungsabstände $\mathrm{K}-\mathrm{N}$ und $\mathrm{K}-\mathrm{O}$ liegen in der Größenordnung von ähnlich aufgebauten, in der Literatur bereits bekannten Systemen ${ }^{3,88,19}$. Untersucht man die Umgebung des niederkoordinierten Metallatoms näher, so findet man intra- als auch intermolekular Kohlenstoff-Kontakte (s. Tab. 3), die jedoch mit Werten zwischen 3.38-3.42 § als äußerst schwache Wechselwirkungen ${ }^{20)}$ einzustufen sind.

Wie in 3 erreichen auch im Kaliumderivat 4 die Stickstoffatome eine vierfache Koordination, während die Sauerstoffatome trigonal planar konfiguriert vorliegen. Ebenso sind in beiden Verbindungen aufgrund der bekannten Polaritäten die $\mathrm{Si}-\mathrm{O}$ - und $\mathrm{Si}-\mathrm{N}$-Bindungen stark verkürzt. Hinzu kommt bei 4 eine noch stärkere Aufweitung des $\mathrm{Si}-\mathrm{N}-\mathrm{Si}$-Winkels. Dieser Effekt kann neben sterischen Gründen (Abstoßung der an den Stickstoff gebundenen Methylsilylgruppen) auch mit dem ionischeren Charakter der Metall-Stickstoffbindung und dem damit vermehrten $s$-Anteil der Bindung zwischen Silicium und Stickstoff (Übergang von $\mathrm{sp}^{3}$ zur $\mathrm{sp}$-Hybridisierung am N-Atom) erklärt werden.

Vergleicht man die Ergebnisse der Strukturanalysen der Verbindungen 3 und $\mathbf{4}$ miteinander, so beobachtet man in Abhängigkeit vom zentralen Alkalimetall unterschiedliche Anordnungen im Festkörper. In der Natriumverbindung 3 findet man zwei- und vierfach koordinierte Metallatome, wohingegen in der Verbindung 4 für die Kaliumatome eine $\lambda^{3}$-Koordination vorliegt. Die unterschiedlichen Strukturen könnten eine Folge der unterschiedlichen Ionenradien und Elektronegativitäten des Natriums und Kaliums ${ }^{21)}$ sein. In diesem Zusammenhang fällt auf, daß auch in basenstabilisierten Lithiumderivaten, die der Verbindung 3 sehr ähnlich sind $^{1,9)}$, solche „Separationen von Metallatomen" vorkommen können. Auch Kristalleffekte sind als Ursachen für die verschiedenen Strukturen von $\mathbf{3}$ und $\mathbf{4}$ nicht auszuschließen.

\section{Experimenteller Teil}

Alle Reaktionen wurden unter Stickstoff als Schutzgas in Stockschen Vakuumapparaturen durchgeführt.

${ }^{1} \mathrm{H}$-NMR-Spektren: (WP80-FT, $80 \mathrm{MHz}$ ), $\delta$-Werte in ppm. Massenspektren: Gerät der Fa. Finnigan MAT 90. - C-, H-, NAnalysen führte die Firma Beller, Göttingen, durch. Alle anderen quantitativen Elementbestimmungen erfolgten auf naßchemischem Wege. - Die Berechnungen zur Strukturbestimmung erfolgten auf 
einer Micro-VAX mit dem SHELX-Programmsystem ${ }^{10}$. Die graphischen Darstellungen wurden mit dem Programmsystem SCHAKAL realisiert ${ }^{22)}$

1-tert-Butoxy-1,1,3,3,3-pentamethyldisilazan (1): $11.0 \mathrm{~g}(0.114 \mathrm{~mol})$ Natrium-tert-butoxid, aufgeschlämmt in $100 \mathrm{ml}$ Diethylether, werden zu einer Lösung von $13 \mathrm{ml}(0.108 \mathrm{~mol})$ Dimethyldichlorsilan in $20 \mathrm{ml}$ Diethylether getropft. Nach $2 \mathrm{~h}$ Kochen unter Rückfluß wird vom Natriumchlorid abfiltriert. In einer Vorlage werden ca. $20 \mathrm{ml}$ Ammoniak einkondensiert, und die etherische Lösung wird langsam zugetropft. Nach Verdampfen des überschüssigen Ammoniaks wird vom unlöslichen Ammoniumchlorid abfiltriert und das Lösungsmittel zum größten Teil über eine Vigreux-Kolonne abdestilliert. Zur verbleibenden Lösung gibt man $100 \mathrm{ml}$ Ether und $15 \mathrm{ml}(0.108 \mathrm{~mol})$ Triethylamin. In diese Lösung tropft man $14 \mathrm{ml}$ (0.11 mol) Chlortrimethylsilan. Nach 90 min Kochen unter Rückfluß wird vom Niederschlag abgetrennt, der Ether über eine Vigreux-Kolonne abdestilliert und das Produkt bei 12 Torr fraktionierend destilliert. Man erhält bei $60-65^{\circ} \mathrm{C} 12.4 \mathrm{~g} \mathrm{(56 \% )} \mathrm{des}$ Aminosilans 1. - ${ }^{1} \mathrm{H}-\mathrm{NMR}$ (Benzol): $\delta=0.14\left[\mathrm{~s} ; 9 \mathrm{H}, \mathrm{Si}\left(\mathrm{CH}_{3}\right)_{3}\right]$, $0.18\left[\mathrm{~s} ; 6 \mathrm{H}, \mathrm{Si}\left(\mathrm{CH}_{3}\right)_{2}\right], 1.25\left[\mathrm{~s} ; 9 \mathrm{H}, \mathrm{OC}\left(\mathrm{CH}_{3}\right)_{3}\right] .-$ IR $\left[\mathrm{cm}^{-1}\right]$ : $v(\mathrm{~N}-\mathrm{H})=3377$; weitere Banden: 2970 (st), $2900(\mathrm{~m}), 1694(\mathrm{w})$, 1456 (w), 1385 (w), 1361 (m), 1254 (st), 1199 (st), 1178 (st), 1051 (st), $1023(\mathrm{~m}), 932$ (st), $876(\mathrm{~m}), 842$ (st), 811 (m), $790(\mathrm{~m}), 754(\mathrm{w})$, $686(\mathrm{w})$.

\section{$\mathrm{C}_{9} \mathrm{H}_{25} \mathrm{NOSi}_{2}$ (219.5) Ber. C 49.25 H 11.48 N 6.38}

Gef. C 50.30 H 11.63 N 6.36

Molmasse 209 (kryoskopisch in Benzol)

Li-Derivat (2): $0.5 \mathrm{~g}$ (2.3 mmol) des Liganden 1 werden in $10 \mathrm{ml}$ Diethylether vorgelegt. Dazu tropft man $2 \mathrm{ml}$ einer $1.6 \mathrm{M}$ Lösung von $n$-BuLi $(3.2 \mathrm{mmol})$ in Hexan. Anschließend wird $2 \mathrm{~h}$ unter Rückfluß gekocht. Nach Abkondensieren des Lösungsmittels wird der Rückstand bei $50^{\circ} \mathrm{C} / 10^{-4}$ Torr sublimiert. Man erhält $0.451 \mathrm{~g}$ (87\%) farbloses, kristallines 2; Schmp. $107^{\circ} \mathrm{C} .-{ }^{1} \mathrm{H}-\mathrm{NMR}$ (Benzol): $\delta=0.27\left[\mathrm{~s} ; 9 \mathrm{H}, \mathrm{Si}\left(\mathrm{CH}_{3}\right)_{3}\right], 0.31\left[\mathrm{~s} ; 6 \mathrm{H}, \mathrm{Si}\left(\mathrm{CH}_{3}\right)_{2}\right], 1.18[\mathrm{~s} ; 9 \mathrm{H}$, $\left.\mathrm{OC}\left(\mathrm{CH}_{3}\right)_{3}\right]$.

$\mathrm{C}_{18} \mathrm{H}_{48} \mathrm{Li}_{2} \mathrm{~N}_{2} \mathrm{O}_{2} \mathrm{Si}_{4}$ (420.8) Ber. C $47.96 \mathrm{H} 10.73 \mathrm{~N} 6.21 \mathrm{Li} 3.08$ Gef. C 48.66 H 10.88 N 6.22 Li 2.58 Molmasse 470 (kryoskopisch in Benzol)

Na-Derivat 3: $0.2 \mathrm{~g}$ (5.1 mmol) Natriumamid werden in $20 \mathrm{ml}$ Toluol vorgelegt. Dazu gibt man $0.8 \mathrm{~g}(3.7 \mathrm{mmol}) 1$. Die Suspension läßt man über Nacht bei Raumtemp. rühren. Danach wird vom überschüssigen Natriumamid abfiltriert, der Niederschlag mit 10 $\mathrm{ml}$ Toluol gewaschen und das Lösungsmittel im Vakuum entfernt. Nach Umkristallisation aus Toluol erhält man $0.778 \mathrm{~g}(86 \%) 3$; Schmp. $210^{\circ} \mathrm{C}$. - Sublimation bei $85^{\circ} \mathrm{C} / 10^{-4}$ Torr. - 'H-NMR (Benzol): $\delta=0.21\left[\mathrm{~s} ; 9 \mathrm{H}, \mathrm{Si}\left(\mathrm{CH}_{3}\right)_{3}\right], 0.30\left[\mathrm{~s} ; 6 \mathrm{H}, \mathrm{Si}\left(\mathrm{CH}_{3}\right)_{2}\right], 1.18[\mathrm{~s} ;$ $\left.9 \mathrm{H}, \mathrm{OC}\left(\mathrm{CH}_{3}\right)_{3}\right]$. - MS (30 eV): Peak höchster Masse: $m / z=482$ $\left(\mathrm{C}_{18} \mathrm{H}_{48} \mathrm{~N}_{2} \mathrm{Na}_{2} \mathrm{O}_{2}{ }^{28} \mathrm{Si}_{4}, \mathrm{M}^{+}\right)$. Kristalldaten von 3 siehe Tab. 1.

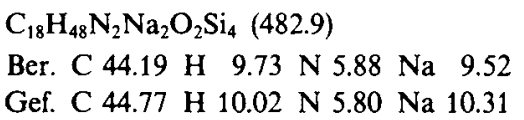

$K$-Derivat 4: Darstellung wie bei 3 beschrieben: ausgehend von $0.130 \mathrm{~g}$ (2.36 mmol) Kaliumamid, $20 \mathrm{ml}$ Toluol, $0.40 \mathrm{~g}(1.82 \mathrm{mmol})$ 1; Ausb. $0.403 \mathrm{~g}(86 \%) 4$; Schmp. $164^{\circ} \mathrm{C}$. - Sublimation bei $120^{\circ} \mathrm{C} /$ $10^{-4}$ Torr. - ${ }^{1} \mathrm{H}-\mathrm{NMR}$ (Benzol): $\delta=0.21\left[\mathrm{~s} ; 9 \mathrm{H}, \mathrm{Si}\left(\mathrm{CH}_{3}\right)_{3}\right], 0.25$ $\left[\mathrm{s} ; 6 \mathrm{H}, \mathrm{Si}\left(\mathrm{CH}_{3}\right)_{2}\right], 1.20\left[\mathrm{~s} ; 9 \mathrm{H}, \mathrm{OC}\left(\mathrm{CH}_{3}\right)_{3}\right]$. - Kristalldaten von 4 sind in Tab. 1 enthalten.

$\mathrm{C}_{18} \mathrm{H}_{48} \mathrm{~K}_{2} \mathrm{~N}_{2} \mathrm{O}_{2} \mathrm{Si}_{4}$ (515.1) Ber. C $41.97 \mathrm{H} 9.39 \mathrm{~N} 5.44 \mathrm{~K} 15.18$ Gef. C 41.73 H 9.66 N 5.32 K 14.82 Molmasse 536 (kryoskopisch in Benzol)
$R b$-Derivat 5: $0.15 \mathrm{~g}(1.76 \mathrm{mmol})$ Rubidium werden in $10 \mathrm{ml}$ Hexan aufgenommen und $0.45 \mathrm{~g}(2.05 \mathrm{mmol}) 1$ zugetropft. Der Mischung werden anschließend $0.22 \mathrm{ml}(1.91 \mathrm{mmol})$ frisch destilliertes Styrol zugesetzt, und es wird anschließend $1 \mathrm{~h}$ auf $50^{\circ} \mathrm{C}$ erhitzt. Danach ist kein elementares Rubidium mehr vorhanden, und beim Abkühlen der Lösung fällt das Produkt als farbloser Feststoff aus. Nachdem das Lösungsmittel vollständig im Vakuum entfernt wurde, läßt sich das Rohprodukt durch Umkristallisation aus Toluol reinigen. Man erhält $0.454 \mathrm{~g} \mathrm{(85 \% )} \mathrm{5;} \mathrm{Schmp.}$ $210^{\circ} \mathrm{C}$. - Sublimation bei $130^{\circ} \mathrm{C} / 10^{-4}$ Torr. - ${ }^{1} \mathrm{H}-\mathrm{NMR}$ (Benzol): $\delta=0.22\left[\mathrm{~s} ; 9 \mathrm{H}, \mathrm{Si}\left(\mathrm{CH}_{3}\right)_{3}\right], 0.26\left[\mathrm{~s} ; 6 \mathrm{H}, \mathrm{Si}\left(\mathrm{CH}_{3}\right)_{2}\right], 1.22[\mathrm{~s} ; 9 \mathrm{H}$, $\left.\mathrm{OC}\left(\mathrm{CH}_{3}\right)_{3}\right]$.

Kristalldaten: $a=9.020(4), b=8.69(1), c=21.18(2) \AA, \beta=$ 91.62(6),$V=1659.9 \AA^{3}$. - Raumgruppe: $P 2_{1} / n$ (isotyp zu 4).

$\mathrm{C}_{18} \mathrm{H}_{48} \mathrm{~N}_{2} \mathrm{O}_{2} \mathrm{Rb}_{2} \mathrm{Si}_{4}(607.9) \quad$ Ber. C $35.57 \mathrm{H} 7.96$ N $4.61 \mathrm{Rb} 28.12$ Gef. C 35.98 H 7.82 N 4.71 Rb 29.50

\section{CAS-Registry-Nummern}

1: 118538-36-6 / 2: 118538-37-7 / 3: 118538-41-3 / 3 (Na-Salz): 118538-38-8 / 4: 118538-42-4 / 4 (K-Salz): 118538-39-9/5: 118538 . 43-5 / 5 (Rb-Salz): $118538-40-2 / \mathrm{Me}_{2} \mathrm{SiCl}_{2}: 75-78-5 / \mathrm{ClSiMe}_{3}: 75-$ 77-4/ Rb: 7440-17-7

"M. Veith, Angew. Chem. 99 (1987) 1; Angew. Chem. Int. Ed. Engl. 26 (1987) 1.

${ }^{2)}$ D. J. Brauer, H. Bürger, G. R. Liewald, J. Organomet. Chem. 308 (1986) 119.

3) M. Veith, R. Rösler, Z. Naturforsch., Teil B, 41 (1986) 1071.

${ }^{4)}$ M. Veith, J. Pöhlmann, $Z$. Naturforsch., Teil B, 43 (1988) 505.

s) J. Pöhlmann, Dissertation, Univ. des Saarlandes, Saarbrücken 1986.

6) M. Veith, F. Goffing, V. Huch, Chem. Ber. 121 (1988) 943.

7) M. F. Lappert, P. D. Power, A. R. Sanger, R. C. Srivastava, Metal and Metalloid Amides, Ellis Horwood, Chichester 1980.

${ }^{8)}$ M. Veith, A. Belo, Z. Naturforsch., Teil B, 42 (1987) 525.

9) M. Veith, J. Böhnlein, V. Huch, Chem. Ber., im Druck.

10) G. M. Sheldrick, Program for Crystal Structure Determination, Version von 1976 und 1986.

11) International Tables for X-ray Crystallography, Bd. IV, Kynoch Press, Birmingham 1974.

12) Weitere Einzelheiten zu den Kristallstrukturbestimmungen können beim Fachinformationszentrum Energie, Physik, Mathematik GmbH, D-7514 Eggenstein-Leopoldshafen 2, unter Angabe der Hinterlegungsnummer CSD-53458, der Autoren und des Zeitschriftenzitats angefordert werden.

13) D. J. Bauer, H. Bürger, W. Geschwandtner, G. R. Liewald, J. Organomet. Chem. 248 (1983) 1.

14) E. Weiss, G. Sauermann, J. Organomet. Chem. 21 (1970) 1.

${ }^{15)}$ R. Grüning, J. L. Atwood, J. Organomet. Chem. 137 (1977) 101.

16) W. Clegg, R. E. Mulvey, R. Snaith, G. E. Toogood, K. Wade, $J$. Chem. Soc., Chem. Commun. 1986, 1740.

17) E. Weiss, H. Alsdorf, Z. Anorg. Allg. Chem. 372 (1970) 206.

18) P. G. Willard, G. B. Carpenter, J. Am. Chem. Soc. 108 (1986) 462 .

19) A. M. Domingos, G. M. Sheldrick, Acta Crystallogr., Sect. B, 30 (1974) 517

20) E. Weiss, G. Sauermann, Chem. Ber. 103 (1970) 265.

21) A. F. Holleman, E. Wiberg, Lehrbuch der Anorganischen Chemie, 91. - 100. Auflage, Walter de Gruyter-Verlag, Berlin-New York 1985.

22) Egbert Keller, SCHAKAL 86, Freiburg 1986.

${ }^{23)}$ W. C. Hamilton, Acta Crystallogr. 12 (1959) 609.

$[287 / 88]$ 